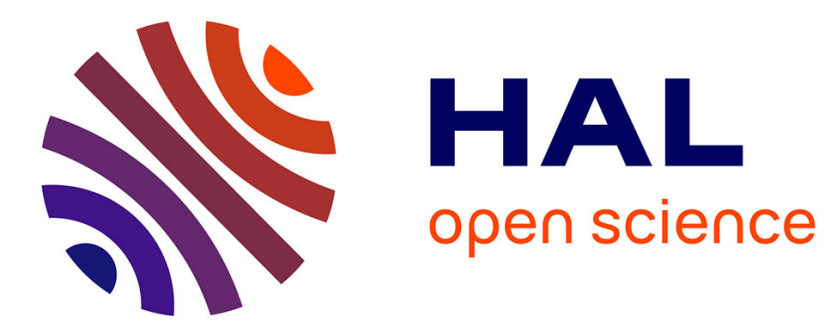

\title{
Structure of the $\mathrm{N}=27$ isotones derived from the ${ }^{44} \mathrm{Ar}(\mathbf{d}, \mathbf{p}){ }^{45} \mathrm{Ar}$
}

L. Gaudefroy, O. Sorlin, F. Nowacki, D. Beaumel, Y. Blumenfeld, Z.

Dombradi, S. Fortier, S. Franchoo, S. Grévy, F. Hammache, et al.

\section{- To cite this version:}

L. Gaudefroy, O. Sorlin, F. Nowacki, D. Beaumel, Y. Blumenfeld, et al.. Structure of the N=27 isotones derived from the ${ }^{44} \mathrm{Ar}(\mathrm{d}, \mathrm{p}){ }^{45} \mathrm{Ar}$. Physical Review C, 2008, 78, pp.034307. 10.1103/PhysRevC.78.034307 . in2p3-00311397

\section{HAL Id: in2p3-00311397 https://hal.in2p3.fr/in2p3-00311397}

Submitted on 26 Aug 2008

HAL is a multi-disciplinary open access archive for the deposit and dissemination of scientific research documents, whether they are published or not. The documents may come from teaching and research institutions in France or abroad, or from public or private research centers.
L'archive ouverte pluridisciplinaire HAL, est destinée au dépôt et à la diffusion de documents scientifiques de niveau recherche, publiés ou non, émanant des établissements d'enseignement et de recherche français ou étrangers, des laboratoires publics ou privés. 


\title{
Study of ${ }^{44} \operatorname{Ar}(d, p){ }^{45} \mathrm{Ar}$ and structure of the $\mathrm{N}=27$ isotones
}

\author{
L. Gaudefroy ${ }^{1,2}$ O. Sorlin, ${ }^{1,3}$ F. Nowacki,${ }^{4}$ D. Beaumel, ${ }^{1}$ Y. Blumenfeld, ${ }^{1}$ Z. Dombrádi, ${ }^{5}$ \\ S. Fortier, ${ }^{1}$ S. Franchoo, ${ }^{1}$ S. Grévy,${ }^{3}$ F. Hammache, ${ }^{1}$ K. W. Kemper,${ }^{6}$ K.L. Kratz,${ }^{7,8}$ \\ M.G.St. Laurent, ${ }^{3}$ S.M. Lukyanov,${ }^{9}$ L. Nalpas, ${ }^{10}$ A.N. Ostrowski, ${ }^{11}$ Yu.-E. Penionzhkevich, ${ }^{9}$ \\ E.C. Pollacco, ${ }^{10}$ P. Roussel,${ }^{1}$ P. Roussel-Chomaz ${ }^{3}$ D. Sohler, ${ }^{5}$ M. Stanoiu,,${ }^{1}$ and E. Tryggestad ${ }^{1}$ \\ ${ }^{1}$ IPN, IN2P3-CNRS,F- 91406 Orsay Cedex, France \\ ${ }^{2}$ CEA/DAM/DIF/DPTA/SPN Bruyères-le-Châtel, 91297 ARPAJON Cedex \\ ${ }^{3}$ Grand Accérateur National d'Ions Lourds (GANIL), CEA/DSM - CNRS-IN2P3, \\ Bd Henri Becquerel, BP 55027, F-14076 Caen Cedex 5, France \\ ${ }^{4}$ IReS, Univ. Louis Pasteur, BP 28, F-67037 Strasbourg Cedex, France \\ ${ }^{5}$ Institute of Nuclear Research, H-4001 Debrecen, Pf. 51, Hungary \\ ${ }^{6}$ Department of Physics, Florida State University, Tallahassee, Florida 32306, USA \\ ${ }^{7}$ Max-Planck-Institut für Chemie, Otto-Hahn-Institut, D-55128 Mainz, Germany \\ ${ }^{8}$ HGF Virtuelles Institut für Struktur der Kerne und Nukleare Astrophysik (VISTARS), D-55128 Mainz, Germany \\ ${ }^{9}$ FLNR/JINR, 141980 Dubna, Moscow region, Russia \\ ${ }^{10}$ CEA-Saclay, DAPNIA-SPhN, F-91191 Gif sur Yvette Cedex, France \\ ${ }^{11}$ Institut für Kernchemie, Universität Mainz, D-55128 Mainz, Germany
}

(Dated: August 26, 2008)

\begin{abstract}
The ${ }^{44} \operatorname{Ar}(\mathrm{d}, \mathrm{p})^{45} \mathrm{Ar}$ neutron transfer reaction was performed at $10 \mathrm{~A} . \mathrm{MeV}$. Measured excitation energies, deduced angular momenta and spectroscopic factors of the states populated in ${ }^{45} \mathrm{Ar}$ are reported. A satisfactory description of these properties is achieved in the shell model framework using a new $s d p f$ interaction. The model analysis is extended to more exotic even- $\mathrm{Z}$ nuclei down to ${ }_{14}^{41} \mathrm{Si}_{27}$ to study how collectivity impacts the low lying structure of $\mathrm{N}=27$ neutron-rich nuclei.
\end{abstract}

PACS numbers: 21.10.Jx, 21.60.Cs, 25.60.-t, 27.40.+z

\section{INTRODUCTION}

The $\mathrm{N}=28$ shell closure has been extensively studied in the last decade via $\beta$-decay measurements [1], Coulomb-excitation [2, 3], gamma- or electron- decay spectroscopy [1, 5, 6, 7, 8, 9, 10, 11, mass measurements 12, 13, and direct reactions [14, 15]. In parallel numerous theoretical efforts have been carried out both in mean field [16, 17, 18, 19] and shell model frameworks 20, 21, 22, 23, 24]. These studies have pointed out that the $\mathrm{Z} \leq 16$ nuclei exhibit collective properties. This feature was ascribed to the erosion of the $\mathrm{N}=28$ shell closure when only four protons are removed from the doubly magic ${ }^{48} \mathrm{Ca}$. The ${ }_{18} \mathrm{Ar}_{N \simeq 28}$ nuclei are therefore turning points to understand the departure from spherical nuclei with single particle states to deformed nuclei. Previous experimental studies on ${ }^{45} \mathrm{Ar}_{27}$ were carried out by means of one-neutron knock-out, $\beta$-decay of ${ }^{45} \mathrm{Cl}$, and in-beam $\gamma$-ray spectroscopy using the fragmentation reaction. The two first experimental techniques were probing the neutron occupied orbitals from which neutrons were either knocked-out or converted into a proton by the weak interaction process. The latter experiment favors the production of Yrast states. It is globally not very selective making the assignment of the excited states difficult. Transfer $(\mathrm{d}, \mathrm{p})$ reaction is a suitable tool to determine the single particle nature of the valence neutron states, as shown for ${ }_{18}^{47} \mathrm{Ar}_{29}$ in Ref. [15]. This method is therefore perfectly adapted to the search for the evolution of the $\mathrm{N}=28$ shell closure. In the present article, we report on the structure of ${ }_{18}^{45} \mathrm{Ar}_{27}$ using the same ex- perimental technique as in [15.

Section II presents a detailed description of the experimental and analysis methods. In section III results of the Distorted Wave Born Approximation (DWBA) analysis of the measured proton differential angular distributions are given. The reported structure of ${ }^{45} \mathrm{Ar}$ is interpreted in the shell model framework in section [V] and the discussion is extended to more exotic $\mathrm{N}=27$ isotones, to study if/how collectivity, observed at $\mathrm{N}=28$, impacts the structure of these nuclei.

\section{EXPERIMENTAL METHOD}

The experiment was performed at the GANIL/SPIRAL facility 25. The ${ }^{44} \mathrm{Ar}$ nuclei were produced by means of the fragmentation of a $66 \mathrm{~A} . \mathrm{MeV}$ ${ }^{48} \mathrm{Ca}$ primary beam into a thick carbon target. The $\mathrm{Ar}$ nuclei were extracted from the target heated at a temperature of $2000^{\circ} \mathrm{K}$ and were purified and accelerated to an energy of $10 \mathrm{~A} \cdot \mathrm{MeV}$ by the CIME cyclotron. For checking and calibration purposes, a stable beam of ${ }^{40} \mathrm{Ar}$ was accelerated to an energy of $11 \mathrm{~A} \cdot \mathrm{MeV}$ in a dedicated setting. The pure argon beams $\left(10^{5} \mathrm{pps}\right.$ in average for both settings) impinged on a $0.38(6) \mathrm{mg} . \mathrm{cm}^{-2}$ thick $\mathrm{CD}_{2}$ target. The angular divergence of the beam was set to a low value of $1.5 \mathrm{mrad}$, as a consequence the FWHM of the beam spot on target was of about $1.5 \mathrm{~cm}$ along both $\mathrm{X}$ and $\mathrm{Y}$ axis. One Multi Wire Proportional Chamber (MWPC) CATS 26] was placed $11 \mathrm{~cm}$ downstream from the target to determine the $(\mathrm{X}, \mathrm{Y})$ position of the reaction 
on target, on an event by event basis. To achieve this goal, the position on CATS was simply translated on the target along the beam axis direction to deduce the vertex localization. The position resolution of the detector was determined in a dedicated run using a mask with calibrated holes located at the target position. Within the experimental conditions, the image of the mask was reconstructed with a resolution of about $1 \mathrm{~mm}$. The MWPC also served to monitor the beam intensity. The efficiency of the MWPC has been determined relative to a plastic scintillator (assumed to be $100 \%$ efficient within the experimental conditions) located behind the CATS detector. The deduced efficiency is $95(5) \%$.

The outgoing proton in the $(\mathrm{d}, \mathrm{p})$ reaction was detected at backward angles in the laboratory frame in one of the 8 modules of the Double Sided Silicon Strip Detector (DSSD) MUST [27. The array was arranged in 2 rings of 4 modules each, located $10 \mathrm{~cm}$ upstream from the target. The internal ring was centered at $110^{\circ}$ in the laboratory frame, and the external one at $145^{\circ}$. The total angular coverage ranged from $95^{\circ}$ to $175^{\circ}$, in the laboratory frame $\left(5^{\circ}<\theta_{\text {c.m. }}<50^{\circ}\right.$, where c.m. stands for center-of-mass). The 3D localization of the detectors was measured with an accuracy of $0.5 \mathrm{~mm}$ by means of a laser positioning device. The geometrical efficiency of the array has been simulated taking into account the measured position of each of the modules with respect to the center of the target. The efficiency curve has a double humped structure (one for each ring) with a maximum efficiency at about 150 degrees, as shown by the set of black points in figure 1 (a). Uncertainties are less than $10 \%$, values which have been determined by shifting the position of the modules by $\pm 0.5 \mathrm{~mm}$ in the simulation. Each module has an active area of $60 * 60 \mathrm{~mm}^{2}$, divided into $60 * 60$ perpendicular strips ( $\mathrm{X}$ and $\mathrm{Y}$ ). The angles of emission of the protons were obtained, with a resolution of one degree in the laboratory frame, from the combination of positions at the target and the MUST array.

Figure 1 (a) also displays the scatter-plot of proton energy versus laboratory angle measured for the ${ }^{44} \operatorname{Ar}(\mathrm{d}, \mathrm{p}){ }^{45} \mathrm{Ar}$ reaction. Kinematic lines are visible corresponding to different final states in ${ }^{45} \mathrm{Ar}$. The weaker statistics in the angular domain around $130^{\circ}$ are due to the low efficiency in this region corresponding to the gap between the external and internal rings. Excitation energies in ${ }^{45} \mathrm{Ar}$ and scattering angles in the center-of-mass frame have been reconstructed using two body kinematics. To reduce the background, the transfer-like residues have been selected and identified by the SPEG spectrometer [28]. The detection system at the focal plane consisted of: i) two Cathode Readout Drift Chambers (CRDC) used for a precise determination of the magnetic rigidity of the transmitted nuclei, ii) an Ionization Chamber (IC) for energy-loss measurement, and iii) a plastic scintillator for the measurement of the time-offlight with respect to the CATS detector and for the determination of the fragment residual energy. To minimize
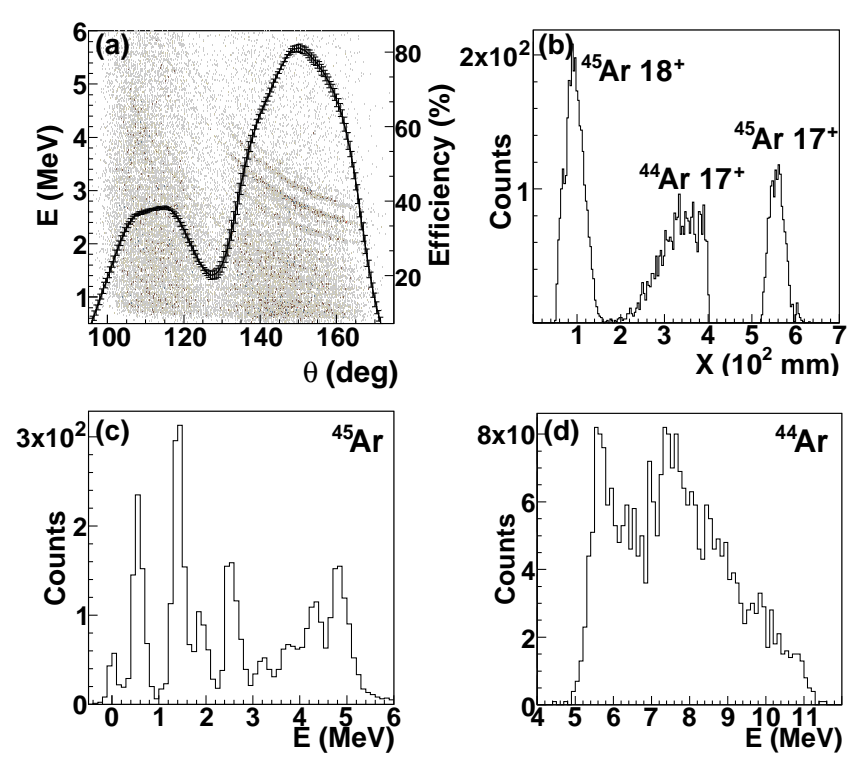

FIG. 1: (a): Kinematic lines of the protons emitted in the ${ }^{44} \mathrm{Ar}(\mathrm{d}, \mathrm{p}){ }^{45} \mathrm{Ar}$ reaction together with geometrical efficiency of the charged particle array (black points). (b) : Position distribution of the reaction residues at the focal plane of SPEG. (c) and (d): Exclusive ${ }^{45}$ Ar excitation energy spectrum, gated on ${ }^{45} \mathrm{Ar}$ and ${ }^{44} \mathrm{Ar}$, respectively (see text).

pile-up in both the CRDC and IC, the elastically scattered nuclei were stopped in a movable active 'finger' of $120 \mathrm{~mm}$ width, placed in front of the detection system.

After traversing the target and CATS detector, the Ar nuclei, initially in the $9^{+}$charge state, were spread over three charge states $18^{+}, 17^{+}$and $16^{+}$, the relative intensities of which have been measured at the SPEG focal plane to be $50 \%, 40 \%$ and $10 \%$, respectively. Due to the limited momentum acceptance of the spectrometer $( \pm 3.5 \%)$, the magnetic rigidity of the spectrometer was set to transmit the two most intense charge states. A typical focal plane position spectrum obtained with the CRDCs is shown for the ${ }^{44} \mathrm{Ar}(\mathrm{d}, \mathrm{p}){ }^{45} \mathrm{Ar}$ reaction in Figure 1 (b). The two peaks at the edges of the focal plane correspond to transmission of ${ }^{45} \mathrm{Ar}$ nuclei in either the $18^{+}$or $17^{+}$ charge state, as labeled on the figure. The bump centered at $350 \mathrm{~mm}$ is due to the detection of ${ }^{44} \mathrm{Ar}$ nuclei originating from the transfer to neutron-unbound states in ${ }^{45} \mathrm{Ar}$. For these neutron break-up events following the (d,p) transfer, only the $17^{+}$charge state was transmitted to the focal plane of the spectrometer. The sharp cut in the focal plane positions between $\mathrm{X}=400 \mathrm{~mm}$ and $\mathrm{X}=520 \mathrm{~mm}$ in Fig. 11 corresponds to the projectile-like particles stopped by the movable finger.

Panels (c) and (d) of Fig. 11 display the ${ }^{45} \mathrm{Ar}$ excitation energy spectra gated on the focal plane position corresponding to transfer towards bound and unbound states, respectively. The neutron separation energy of ${ }^{45} \mathrm{Ar}$ is $5.2 \mathrm{MeV}$. Fig. 2 (a) shows the inclusive excitation energy spectrum for ${ }^{45} \mathrm{Ar}$, reconstructed using only the MUST and CATS detectors. As compared to the ex- 


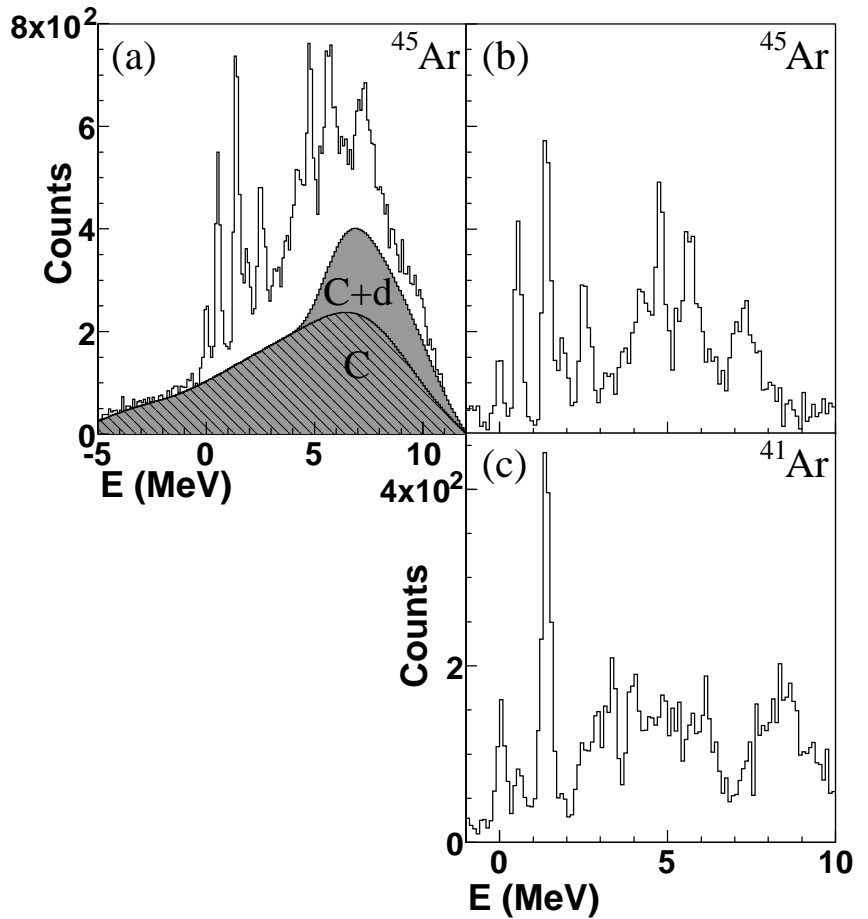

FIG. 2: (a): Inclusive excitation energy spectrum of ${ }^{45} \mathrm{Ar}$. The dashed grey area (labeled C) corresponds to the measured carbon-induced background. The grey area $(\mathrm{C}+\mathrm{d})$ is the sum of the carbon and deuteron break-up -induced backgrounds. (b) and (c): Background subtracted inclusive excitation energy spectra for the ${ }^{45} \mathrm{Ar}$ and ${ }^{41} \mathrm{Ar}$ nuclei, respectively.

clusive spectra of Figs. 1 1 (c) and 1 1 (d) the inclusive spectrum exhibits background contributions arising from the deuteron break-up and from reactions with the carbon nuclei contained in the $\mathrm{CD}_{2}$ target that are removed when the coincidence with a transfer-like product in SPEG is imposed. However, a loss of about a factor of 2 in transmission is found, attributed to optical aberrations at the very edges of the focal plane, where the transfer-like nuclei are located. Therefore the exclusive spectrum was used only to ascertain the origin of all the peaks observed in the inclusive data, for which the two estimated background components have been subtracted.

The carbon-induced background has been determined in a dedicated run using a pure carbon target. This component, normalized to the number of $\mathrm{C}$ nuclei in the $\mathrm{CD}_{2}$ target and the number of incident particles, accounts for the negative excitation energy part of the ${ }^{45} \mathrm{Ar}$ spectrum presented in Fig. 2 (a). The deuteron breakup induced background has been determined through a Monte-Carlo phase-space calculation giving the relation between the proton energies and angles [29]. The amplitude of this contribution was normalized to reproduce the high-energy tail of the inclusive spectrum of Fig. 2 (a). The corresponding background subtracted spectrum is presented on Figure 2 (b) for ${ }^{45} \mathrm{Ar}$. A similar procedure has been applied to the case of ${ }^{41} \mathrm{Ar}$, leading to the excitation energy spectrum presented on Figure 2 (c).
TABLE I: Excitation energies $\left(\mathrm{E}^{*}\right)$ in $\mathrm{keV}$, angular momenta $(\ell)$ and vacancy values $\left([\mathrm{J}] \cdot \mathrm{C}^{2} \mathrm{~S}\right)$ deduced from the present experiment for ${ }^{41,45} \mathrm{Ar}$, compared to previous experimental results from a: Ref. 30, b: Ref. 31], c: Ref. [0], d: Ref. [32], e: Ref. [14]. For previous work the spin/parity $\left[(2 \mathrm{~J})^{\pi}\right]$ is also reported when available. The symbol $[\mathrm{J}]$ means $(2 \mathrm{~J}+1)$.

\begin{tabular}{|c|c|c|c|c|}
\hline \multicolumn{3}{|c|}{ Previous work } & \multicolumn{2}{|c|}{ Present work } \\
\hline $\mathrm{E}^{*}$ & $\ell,(2 \mathrm{~J})^{\pi}$ & {$[\mathrm{J}] \cdot \mathrm{C}^{2} \mathrm{~S}$} & $\mathrm{E}^{*}$ & $\ell[\mathrm{J}] \cdot \mathrm{C}^{2} \mathrm{~S}$ \\
\hline \multicolumn{5}{|c|}{${ }^{41} \mathbf{A r}_{23}$} \\
\hline 0 & $3,7^{-}$ & $3.12^{a}, 4.24(72)^{b}$ & $0(60)$ & $32.96(48)$ \\
\hline $516(2)$ & $1,3^{-}$ & $0.28^{a}, 0.36(8)^{b}$ & $575(60)$ & $10.32(8)$ \\
\hline $1357(2)$ & $1,3^{-}$ & $1.48^{a}, 1.68(32)^{b}$ & $1360(60)$ & $11.92(36)$ \\
\hline $2398(3)$ & $1,1^{-}$ & $0.26^{a}, 0.30(6)^{b}$ & $2435(70)$ & \\
\hline $3327(4)$ & $1,1^{-}$ & $0.58^{a}, 0.72(12)^{b}$ & $3320(60)$ & $10.58(12)$ \\
\hline $3968(2)$ & $1,1^{-}$ & $0.94^{a}, 0.80(14)^{b}$ & $3970(60)$ & $10.72(14)$ \\
\hline \multicolumn{5}{|c|}{${ }^{45} \mathbf{A r}_{27}$} \\
\hline $0^{c, d, e}$ & $3,7^{-}$ & & $0(60)$ & $31.52(40)$ \\
\hline $535(5)^{c, d, e}$ & $1,3^{-}$ & & $550(60)$ & $10.76(20)$ \\
\hline $1415(10)^{d, e}$ & & & $1420(60)$ & $11.08(16)$ \\
\hline $1765(14)^{c}$ &,$-(1,3)^{-}$ & & $1880(60)$ & $10.32(2)$ \\
\hline & & & $2510(60)$ & $10.46(6)$ \\
\hline & & & $3230(60)$ & \\
\hline & & & $3720(85)$ & \\
\hline & & & $4280(65)$ & \\
\hline & & & $4770(60)$ & $\begin{array}{ll}3 & 1.08(24)\end{array}$ \\
\hline & & & $4770(60)$ & $42.10(40)$ \\
\hline & & & $5630(115)$ & 3 1.14(18) \\
\hline & & & $5630(115)$ & $42.20(40)$ \\
\hline & & & $7290(60)$ & \\
\hline
\end{tabular}

\section{RESULTS}

In this section, excitation energies of the states populated in ${ }^{41} \mathrm{Ar}$ and ${ }^{45} \mathrm{Ar}$ are reported, as well as the transferred angular momenta and spectroscopic factors resulting from the DWBA analysis of the proton differential angular cross sections. Details on the uncertainties arising from the analysis procedure are also provided.

A global fit of the spectra displayed in Figs. 2 (b) and 2 (c) has been preformed with a sum of gaussians, taking a common Full Width Half Maximum of $\mathrm{FWHM}=280 \mathrm{keV}$ for each peak. The mean excitation energies resulting from the fit are reported in Table $[$. Eleven states have been identified in ${ }^{45} \mathrm{Ar}$, seven of which for the first time. Quoted errors on excitation energies include statistical errors, propagated uncertainty on the energy calibration of the DSSDs $(\simeq 30 \mathrm{keV})$, and the error arising from the uncertainty on the reconstructed angle of emission of the proton $(\simeq 50 \mathrm{keV})$. To minimize this latter contribution to the total uncertainty, the positions of the DSSDs have been constrained using the data obtained on the transfer reaction with the stable ${ }^{40} \mathrm{Ar}$ beam. The two first excited states in ${ }^{45} \mathrm{Ar}$ [5] as well as the Q-values for the transfer reaction with both ${ }^{40,44} \mathrm{Ar}$ beams [33] also served for this purpose, since 


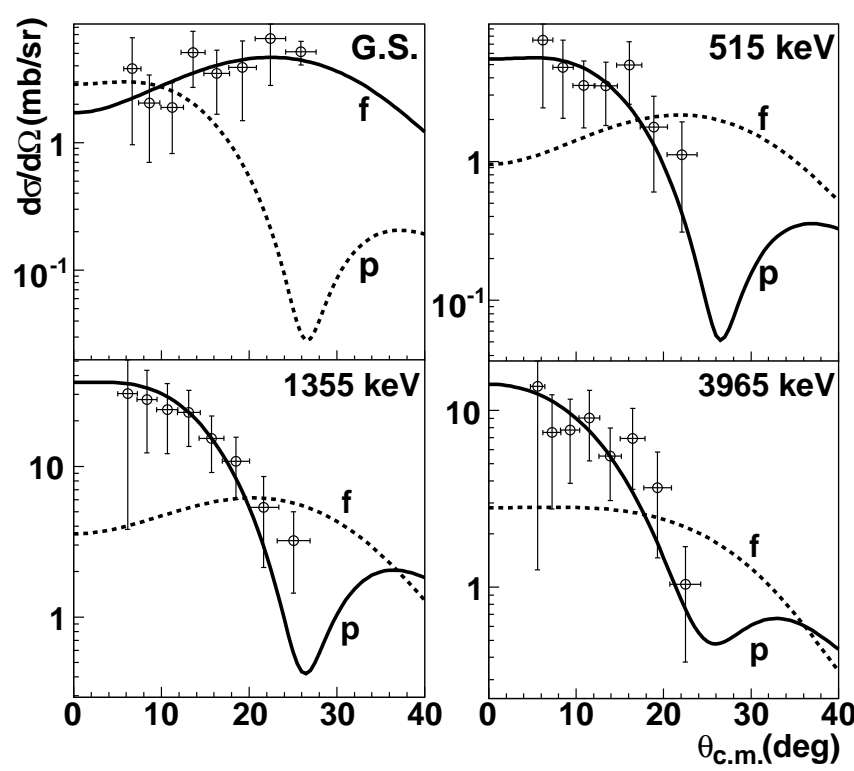

FIG. 3: Proton angular distributions, in the center-of-mass system, of the states populated in ${ }^{41} \mathrm{Ar}$, labeled by their excitation energies. DWBA curves, for $\ell=1(p)$ or $\ell=3(f)$ units of transferred angular momenta, are fitted to the data points.

they were known prior to this experiment, with good accuracy (about $10 \mathrm{keV}$ for the worst case).

The excitation energy spectra of ${ }^{41,45} \mathrm{Ar}$ have been divided in $6^{\circ}$ wide slices (in the laboratory frame) to deduce the differential cross sections of the populated states. The uncertainties on these cross sections arise from statistics, background normalization, thickness of the $\mathrm{CD}_{2}$ reaction target, incident number of particles, and geometrical efficiency. The total uncertainty for each point of the differential cross sections presented in Figs. 3 and 1 is dominated by statistical error. DWBA calculations have been performed using the DWUCK4 code [34], assuming $\ell=1,3$ and 4 units of transferred angular momenta for each significantly populated state in the neutron transfer reaction. Given the limitations of the present work (beam intensity of about $10^{5} \mathrm{pps}$ and 53.5 hours beam time), the minimum measurable differential cross section is of the order of $1 \mathrm{mb} . s r^{-1}$. Global optical potentials, especially suited for the studied mass region and energy of the reactions, have been used for both entrance, $\left(\mathrm{d}+{ }^{40,44} \mathrm{Ar}\right)$ [35, 36], and exit, $\left(\mathrm{p}+{ }^{41,45} \mathrm{Ar}\right)$ [37, 38] channels. The neutron form factor was calculated using a Woods-Saxon shaped potential, parameters of which were adjusted to reproduce the binding energy and quantum numbers of the populated state. The DWBA predictions were fitted to the experimental data points, allowing to deduce: i) the transferred angular momentum from the shape of the distribution, and ii) the spectroscopic factors from the relative normalization between the calculation and the data points. Results of the fit are displayed on figures 3 and 4 ; the presented DWBA curves being

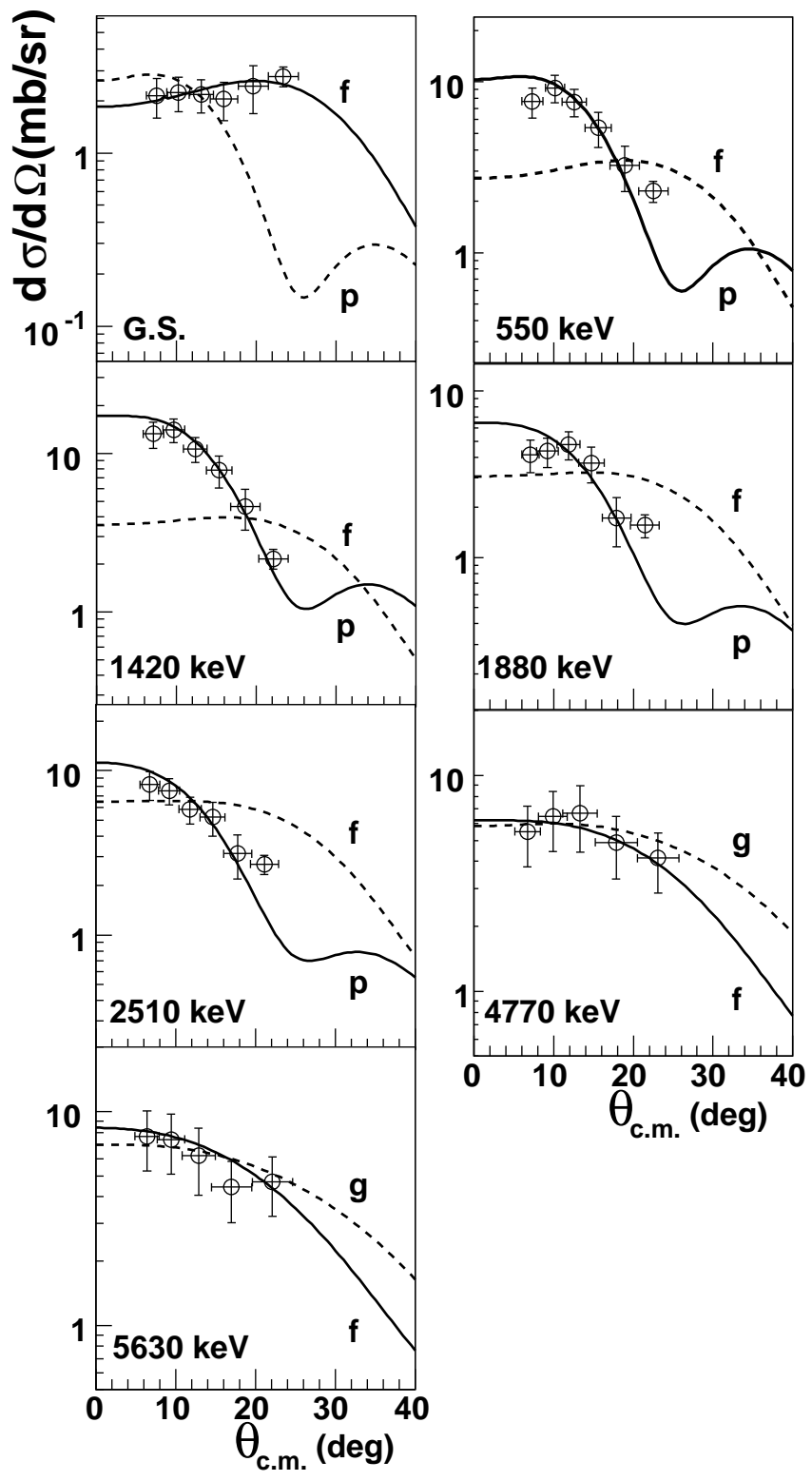

FIG. 4: Same as Fig. 3, for ${ }^{45}$ Ar. $\ell=4$ units of transferred angular momentum $(g)$ is also considered.

calculated with the potentials from Refs. [35] and [37]. Results for ${ }^{41} \mathrm{Ar}$ are compatible with previously reported work, ascertaining the present experimental and analysis procedure. For all significantly populated states, except the ones at $4.7 \mathrm{MeV}$ and $5.6 \mathrm{MeV}$ in ${ }^{45} \mathrm{Ar}$, the angular momentum is unambiguously attributed. The shapes of the angular distribution corresponding to $\ell=3$ and 4 units of transferred momentum are too similar to be disentangled for the 4.7 and $5.6 \mathrm{MeV}$ states in ${ }^{45} \mathrm{Ar}$.

Spectroscopic factors are found to vary within $15 \%$, depending on the set of optical potential used in the DWBA calculations, while the shapes of the distributions are independent of this choice. In Table If, the vacancy values, corresponding to the product of the 
deduced spectroscopic factors by the spin factor $2 J+1$ (noted as $[\mathrm{J}] \cdot \mathrm{C}^{2} \mathrm{~S}$ ), are the mean values deduced from adjustments using the four sets of potentials.

\section{DISCUSSION}

This section aims at determining how the onset of collectivity impacts the low-lying structure of $\mathrm{N}=27$ isotones from ${ }_{20} \mathrm{Ca}$ to ${ }_{14} \mathrm{Si}$. The known low-lying structure of ${ }^{47} \mathrm{Ca}$, and that reported here on ${ }^{45} \mathrm{Ar}$ will serve as anchors to discuss the structure of $\mathrm{S}$ and $\mathrm{Si}$ isotones. In the Shell Model (SM) framework, collectivity is equivalent to the superposition of several particle-hole $(p h)$ components beyond the assumed close core in the Wave Functions (WFs) of the states of interest. Therefore an original representation of the WFs of the calculated states in terms of proton $\left(N_{\pi}\right)$ and neutron $\left(N_{\nu}\right)$ patricle-hole $(p h)$ configurations will serve to determine the degree of collectivity of the nuclei under consideration.

The experimental data on ${ }^{47} \mathrm{Ca}$ and ${ }^{45} \mathrm{Ar}$ are compared to SM calculations performed with the ANTOINE code [39, 40] using a modified version of the $s d f p$ interaction 141. A detailed discussion of these modifications, leading to the SDPF-NR interaction, is presented in Ref. 42]. The full $s d(f p)$ valence space was considered for proton (neutron) excitations. Using such a valence space for studying the nuclei of interest is valid under the reasonable assumption that the $\mathrm{Z}=20$ and $\mathrm{N}=20$ gaps preclude nucleon excitations from $s d$ to $f p$ orbits. In ${ }^{47} \mathrm{Ca}$, the $s d$ shell is completely filled. Therefore only 0 particle 0 hole $(0 p 0 h)$ proton configuration exists within this valence space. With respect to a ${ }^{48} \mathrm{Ca}$ core, the ground state configuration of ${ }^{47} \mathrm{Ca}$ is mainly by the $0 p 1 h$ neutron configuration. It corresponds to a hole in the neutron $\nu f_{7 / 2}$ orbit, and no particle in the $\nu p_{3 / 2}$ and upper orbits. For lighter $\mathrm{Z}$ nuclei, protons excitations within the $s d$ shells are allowed, together with neutron excitations within the $f p$ shells. Proton excitations of even- $Z$ nuclei follow the general form:

$$
N_{\pi} p\left(N_{\pi}\right) h, N_{\pi}=0,1,2 \ldots
$$

In a similar manner, neutron excitations of odd-N nuclei are expressed as:

$$
N_{\nu} p\left(N_{\nu}+1\right) h, N_{\nu}=0,1,2 \ldots
$$

These expressions will be used extensively and commented in the following discussion.

In ${ }^{47} \mathrm{Ca}$ the measured spectroscopic factors of the ground state and first excited state at $2.02 \mathrm{MeV}$ [43] contain more than $80 \%$ of the sum rule limit for the neutron single particle $f_{7 / 2}$ and $p_{3 / 2}$ states, respectively. As said earlier the $7 / 2_{1}^{-}$ground state corresponds to a $0 p 1 h$ neutron configuration, where a single hole remains in the $\nu f_{7 / 2}$ orbit. The $3 / 2_{1}^{-}$first excited state has an almost pure $1 p 2 h$ neutron configuration, with a single neutron

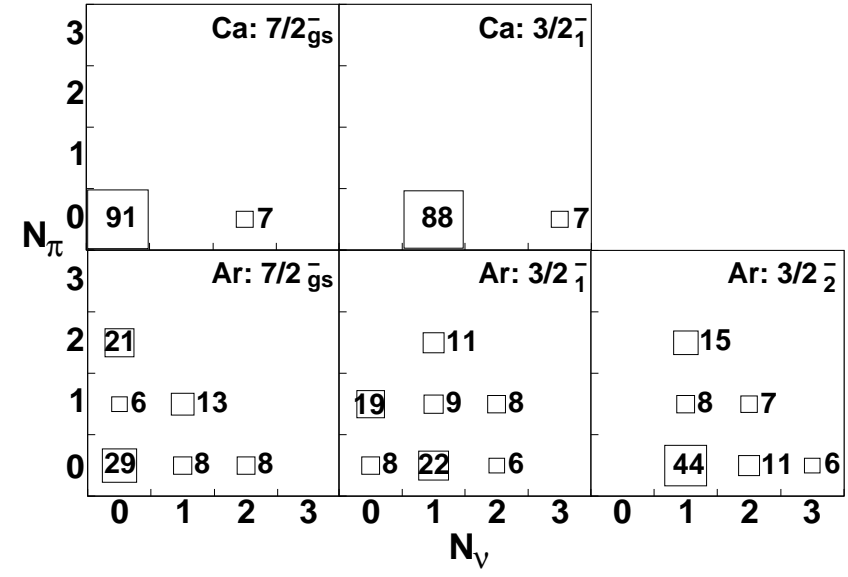

FIG. 5: Squared WF of the first $7 / 2^{-}$and $3 / 2^{-}$states in ${ }^{47} \mathrm{Ca}$ (upper panels) and ${ }^{45} \mathrm{Ar}$ (lower panels) isotones, in the proton $\left(N_{\pi}\right)$ versus neutron $\left(N_{\nu}\right)$ particle-hole configuration plane (see text for details). Excitation energies of the states are given in the text.

promoted into the $\nu p_{3 / 2}$ orbit and two remaining holes in the $\nu f_{7 / 2}$ one. In the SM framework this state is calculated at $2075 \mathrm{keV}$, in good agreement with experiment. The calculated WFs of the $7 / 2_{\text {gs }}^{-}$and $3 / 2_{1}^{-}$states in ${ }^{47} \mathrm{Ca}$ are presented in the two-dimensional plot displayed in Fig. 5. On this figure, each panel represents the WF of a state in the considered nucleus, as labelled on the figure. The WF of each state is decomposed in terms of its proton $\left(N_{\pi}\right.$ on the $\mathrm{Y}$ axis $)$ and neutron $\left(N_{\nu}\right.$ on the $\mathrm{X}$ axis) ph configurations. The percentage of the WF for a couple of $\left(N_{\pi}-N_{\nu}\right)$ values is proportionnal to the area of the box centred at $\left(N_{\pi}-N_{\nu}\right)$. Only the percentages larger than $5 \%$ have been reported on the figure. For the sake of precision each percentage is indicated near its corresponding box. As seen from the first row of Fig. 5, the calculated WFs of the $7 / 2_{1}^{-}$and $3 / 2_{1}^{-}$states in ${ }^{47} \mathrm{Ca}$ exhibit quasi-pure configurations with $N_{\nu}=0(0 p 1 h)$ and $N_{\nu}=1(1 p 2 h)$, respectively. We remind that, being restricted to the $s d$ valence space, the only possible proton configuration in ${ }^{47} \mathrm{Ca}$ is $N_{\pi}=0(0 p 0 h)$.

As compared to ${ }_{20}^{47} \mathrm{Ca}_{27}$, the ${ }_{18}^{45} \mathrm{Ar}_{27}$ nucleus has a more complex low-lying structure, mainly due to the fact that protons excitations are not hindered anymore. Five states have been found in the present experiment below an excitation energy of $2.5 \mathrm{MeV}$, as shown in Fig. 6 The states discovered in Refs. [5, 14, are labeled by a star $\left(^{*}\right)$ in this figure. The agreement between experimental and calculated excitation energies, angular momenta and spectroscopic factors is satisfactory. Minor discrepancies on spectroscopic factors exist for the states arising from re-couplings to core excitation (indicated by dashed lines in the SM level scheme on Fig. 6). Coupled channels reaction calculations could provide better description of the measured differential cross sections of these peculiar states. Such a treatment is left for future work. The angular momentum of the ground state is $\ell=3$, whereas the 


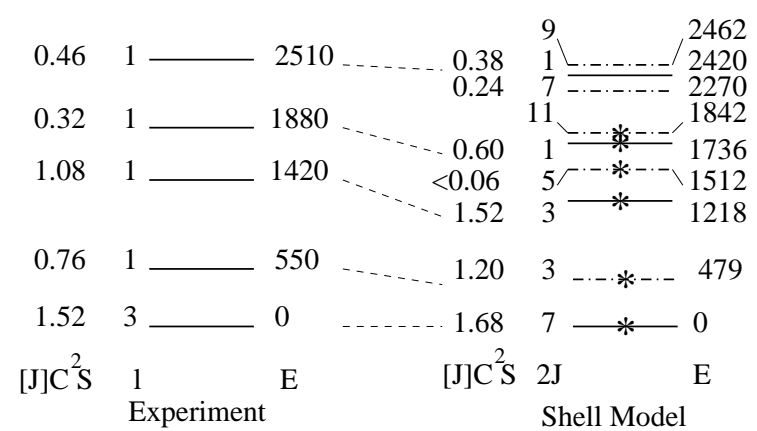

FIG. 6: Experimental level scheme of ${ }^{45} \mathrm{Ar}$, deduced from the present work, compared with SM calculation. States are labeled by their excitation energies $(\mathrm{E})$ in $\mathrm{keV}$, orbital angular momentum (l), spin (2J) and vacancy values $\left([\mathrm{J}] \cdot \mathrm{C}^{2} \mathrm{~S}\right.$, with $[\mathrm{J}]=(2 \mathrm{~J}+1))$. The calculated levels shown as dashed lines are identified as core excited states (see text). Experimental levels observed in previous works 1 , 14 are tentatively identified with the shell model levels marked with stars $\left(^{*}\right)$.

next four other states have $\ell=1$. None of the reported spectroscopic factors of these $\ell=1$ states exhausts the corresponding single-particle strength. This picture contrasts drastically with the one in ${ }^{47} \mathrm{Ca}$. This comparison already points towards an enhancement of configuration mixing below the $\mathrm{Z}=20$ shell, as decribed in the following.

The mixing of configurations is clearly seen while looking at the WFs of the first three states of ${ }^{45} \mathrm{Ar}, 7 / 2_{g s}^{-}, 3 / 2_{1}^{-}$ and $3 / 2_{2}^{-}$, displayed in lower panels of Fig. 5 . The proton and neutron excitations reported on the figure for ${ }^{45} \mathrm{Ar}$ are built on the following reference configuration: $\left(\pi d_{5 / 2}\right)^{6}\left(\pi s_{1 / 2}\right)^{2}\left(\pi d_{3 / 2}\right)^{2}-\left(\nu f_{7 / 2}\right)^{8}$. The WFs of these states span over several neutron and proton configurations in the $\left(N_{\nu}, N_{\pi}\right)$ particle-hole representation. The neutron configuration of the $7 / 2_{g s}^{-}$is peaked at $N_{\nu}=0$ $(0 p 1 h)$, which corresponds to a neutron $f_{7 / 2}$ hole configuration. As the proton $\pi s_{1 / 2}$ and $\pi d_{3 / 2}$ orbits are quasi-degenerate in energy 44, pairing-like excitations are also favored. This explains the equivalent partition of the WF between $N_{\pi}=0$ and $N_{\pi}=2$ proton configurations. Additionally $13 \%$ of the WF is present at $\left(N_{\nu}=1, N_{\pi}=1\right)$. It corresponds to a configuration where the $\nu p_{3 / 2}$ unpaired neutron couples to a $2^{+}$state built on the proton $1 p 1 h\left[\left(\pi s_{1 / 2}\right)^{1}\left(\pi d_{3 / 2}\right)^{3}\right]$ quadrupole coupling.

As for ${ }^{47} \mathrm{Ca}$, a $3 / 2^{-}$excited state with an $\left(N_{\nu}=1\right.$, $\left.N_{\pi}=0\right)$ configuration is expected in ${ }^{45} \mathrm{Ar}$. From Fig. 5 , it is seen that the $3 / 2_{2}^{-}$state at $1.42 \mathrm{MeV}$ is likely to corresponds to the $3 / 2_{1}^{-}$state at $2 \mathrm{MeV}$ in ${ }^{47} \mathrm{Ca}$, as their wave functions are peaked at a similar neutron configuration. When summed over the corresponding proton $p h$ configurations $\left(N_{\pi}=0,1\right.$ and 2 in Fig. 5) $)$, about $70 \%$ of the WF of the $3 / 2_{2}^{-}$state in ${ }^{45} \mathrm{Ar}$ is exhausted by the $N_{\nu}=0$ configuration. It makes the link between this latter state and the $3 / 2_{1}^{-}$state in ${ }^{47} \mathrm{Ca}$ more apparent.

Conversely, the $3 / 2-$ state in ${ }^{45} \mathrm{Ar}$ at $550 \mathrm{keV}$ exhibits

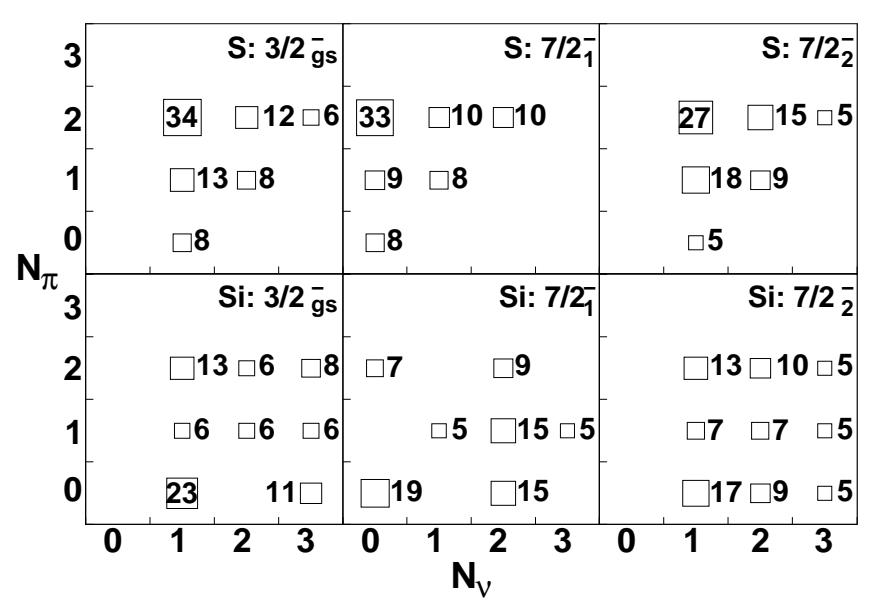

FIG. 7: Same as Fig. 目 for ${ }^{43} \mathrm{~S}$ and ${ }^{41} \mathrm{Si}$ nuclei.

a complex superposition of various values of $N_{\pi}$ and $N_{\nu}$ ranging from 0 to 2 . For this state one can however distinguish equal contributions (about $20 \%$ as seen on Fig.5) of $\left(N_{\nu}=1, N_{\pi}=0\right)$ and $\left(N_{\nu}=0, N_{\pi}=1\right)$ configurations. The former configuration resembles the one of the $3 / 2_{2}^{-}$state, whereas the second is due to the coupling of a $\nu f_{7 / 2}$ hole with the $2^{+}$built on proton excitations. This $3 / 2_{1}^{-}$state has no counterpart in the low energy spectrum of ${ }^{47} \mathrm{Ca}$, as proton excitations are weakened there. The strong configuration mixing found in the present SM calculations accounts for the low excitation energy of this $3 / 2^{-}$state in ${ }^{45} \mathrm{Ar}$.

Other components of the multiplet originating from the $\nu f_{7 / 2} \otimes 2^{+}$re-coupling $(J=5 / 2,7 / 2,9 / 2,11 / 2)$ have a negligible single-particle character, contrary to the $3 / 2_{1}^{-}$ state. Therefore these states (indicated with dashed lines in Fig. 6) are not populated by the stripping $(\mathrm{d}, \mathrm{p})$ reaction. Rather good candidates for the $5 / 2^{-}$and $11 / 2^{-}$ states of the multiplet are reported in Ref. [5] using inbeam $\gamma$-spectroscopy following projectile fragmentation. The predicted $\ell=1, \mathrm{~J}^{\pi}=1 / 2^{-}$states are also seen in the present experimental spectrum, at $1.88 \mathrm{MeV}$ and $2.51 \mathrm{MeV}$. Their WFs show a mixing of the $\nu p_{1 / 2}$ singleparticle component (about $20 \%$ of the WFs) and the $\nu p_{3 / 2} \otimes 2^{+}$re-coupling (about $50 \%$ of the WFs). This explains why their spectroscopic factors are not close to unity, as it should be for unmixed states.

Shell model calculations have been undertaken for the ${ }^{43} \mathrm{~S}$ and ${ }^{41} \mathrm{Si}$ nuclei as well. The $\mathrm{WFS}$ for the first $3 / 2_{1}^{-}$ and $7 / 2_{1,2}^{-}$states are shown in Fig. 7. For ${ }^{43} \mathrm{~S}$, the reference configuration on which the $p h$ configurations, reported in Fig. 7, are built is $\left(\pi d_{5 / 2}\right)^{6}\left(\pi s_{1 / 2}\right)^{2}-\left(\nu f_{7 / 2}\right)^{8}$. For ${ }^{41} \mathrm{Si}$ the reference configuration is $\left(\pi d_{5 / 2}\right)^{6}-\left(\nu f_{7 / 2}\right)^{8}$. In $\mathrm{SM}$ calculations, the excitation energies of the states shown in Fig. 7 for ${ }^{43} \mathrm{~S}$ are: $\mathrm{E}\left(7 / 2_{1}^{-}\right)=700 \mathrm{keV}$ and $\mathrm{E}\left(7 / 2_{2}^{-}\right)=950 \mathrm{keV}$. These values have to be compared to the experimental ones: $\mathrm{E}\left(7 / 2_{1}^{-}\right)=319(2) \mathrm{keV}$ [12] and $\mathrm{E}\left(7 / 2_{2}^{-}\right) \simeq 940 \mathrm{keV}$ [3]. No experimental data are available for ${ }^{41} \mathrm{Si}$. The states shown in Fig. 7 for 
this nucleus are calculated at $\mathrm{E}\left(7 / 2_{1}^{-}\right)=140 \mathrm{keV}$ and $\mathrm{E}\left(7 / 2_{2}^{-}\right)=875 \mathrm{keV}$.

At first glance, it is seen that the configuration mixing is growing further in the lighter $\mathrm{N}=27$ isotones. Even if highly mixed in both cases, the structure of the $\mathrm{S}$ and $\mathrm{Si}$ isotones differs noticeably. In the ${ }^{43} \mathrm{~S}$ nucleus proton excitations within the $s_{1 / 2}-d_{3 / 2}$ doublet of orbits easily develop, as witnessed by the peaked WF at $N_{\pi}=2$. Conversely proton excitations are more hindered in ${ }^{41} \mathrm{Si}$, as they require to cross the $\mathrm{Z}=14$ shell gap. As discussed in Ref. 45], proton excitations are weaker in Si than $\mathrm{S}$ chain but must be present to account for the low $2^{+}$ energy of ${ }^{42} \mathrm{Si} 11$. All states shown in ${ }^{41} \mathrm{Si}$ exhibit a larger range of neutron configuration as compared to the heavier isotones. This was ascribed to the global shrink in energy of all neutron $f p$ orbits between $\mathrm{Z}=20$ and $\mathrm{Z}=1411$.

Noteworthy is the inversion between the natural neutron $0 p 1 h\left(N_{\nu}=0\right)$ and the $1 p 2 h\left(N_{\nu}=1\right)$ configurations that is occuring below ${ }^{45} \mathrm{Ar}$. The $3 / 2_{1}^{-}$-like state in ${ }^{45} \mathrm{Ar}$ is becoming the ground state in ${ }^{43} \mathrm{~S}$ and ${ }^{41} \mathrm{Si}$. For ${ }^{43} \mathrm{~S}$, Sarazin et al. 12 evidenced the existence of an isomeric state at $319(2) \mathrm{keV}$, the decay of which was already interpreted as a $7 / 2_{1}^{-}$to the $3 / 2_{1}^{-}$ground state transition. To obtain a deeper understanding of the origin of this inversion, monopole- and multipole-energy contributions to the total energies of the states of interest have been extracted. The monopole energy represents the mean field contribution, of spherical Hartree-Fock type, to the total energy of the state. As demonstrated in Ref. [46], multipole energy corresponds to correlation contributions. The $7 / 2-$ state is calculated as the ground state of each studied $\mathrm{N}=27$ isotone at the monopole level. The multipole energy is found to be about $2 \mathrm{MeV}$, $9 \mathrm{MeV}, 12 \mathrm{MeV}$ and $15 \mathrm{MeV}$ in $\mathrm{Ca}, \mathrm{Ar}, \mathrm{S}$ and Si isotones, respectively. This increase of correlation energy along $\mathrm{N}=27$ favours configuration mixing as shown in Figs. 5 and 7. The energy decomposition also shows that the $3 / 2{ }_{1}^{-}$states in ${ }^{43} \mathrm{~S}$ and ${ }^{41} \mathrm{Si}$ are energetically lowered by about $2 \mathrm{MeV}$, with respect to the $7 / 2_{1}^{-}$state, because of a multipole energy gain in the former state. It thus appears that the increase of correlation energies is mainly responsible for the evolution of the low lying structure along $\mathrm{N}=27$. Similar results on the role of correlations at the $\mathrm{N}=28$ shell closure can be found in Ref. 223. which used an older version of the $s d p f$ interaction 41 .

\section{SUMMARY}

The spectroscopy of ${ }^{45} \mathrm{Ar}$ has been studied through the $\mathrm{d}\left({ }^{44} \mathrm{Ar}, \mathrm{p}\right){ }^{45} \mathrm{Ar}$ transfer reaction performed at $10 \mathrm{~A} . \mathrm{MeV}$. Excitation energies of eleven states, up to $7.3 \mathrm{MeV}$, have been reported, seven of which for the first time. Orbital angular momenta and spectroscopic factors of the most strongly populated states have been deduced from DWBA analysis of the measured proton angular distributions. Below 4.5 MeV, the deduced angular momenta are in agreement with neutron excitations restricted to the $f p$ shell. At higher excitation energies, measured angular distributions are compatible with $\ell=4$ units of transferred angular momentum. The present data have been successfully interpreted in the shell model framework, using a new $s d p f$ interaction. Core excitations are found to significantly fragment the spectroscopic strength for the low lying states in ${ }^{45} \mathrm{Ar}$. The theoretical study has been extended to other $\mathrm{N}=27$ isotones ranging from $\mathrm{Ca}$ to Si. Erosion of the $\mathrm{N}=28$ spherical gap and increase of correlation energies have been shown to account for the development of configuration mixing while going away from the $\beta$-stability line. The $1 p 2 h$ neutron component is found to be the ground state in both ${ }^{43} \mathrm{~S}$ and ${ }^{41} \mathrm{Si}$ instead of the natural $0 p 1 h$ configuration because of the gain of correlation energy in the former configuration.

\section{Acknowledgments}

L.G. thanks J. P. Delaroche, S. Péru and J. F. Berger for fruitful and enlightening discussions. K.-L.K acknowledges financial support from Helmholtz Gemeinschaft under grant VH-VI-061, and from Deutsche Forschungsgemeinschaft (DFG) under contract KR 806/13-1. K.W.K. was supported by the US National Science Foundation and the State of Florida.
[1] O. Sorlin et al., Phys. Rev. C 47, 2941 (1993)

[2] H. Scheit et al., Phys. Rev. Lett. 77, 3967 (1996)

[3] R. W. Ibbotson, T. Glasmacher, P. F. Mantica and H. Scheit, Phys. Rev. C 59, 642 (1999)

[4] D. Sohler et al., Phys. Rev. C 66, 054302 (2002)

[5] Zs. Dombrádi et al., Nucl. Phys. A727, 185 (2003)

[6] O. Sorlin et al., Eur. Phys. J. A22, 173 (2004)

[7] A. Gade et al., Phys. Rev. C 74, 034322 (2006)

[8] J. Fridmann et al., Phys. Rev. C74, 034313 (2006)

[9] S. Grévy et al., Phys. Lett. B 594, 252 (2004)

[10] S. Grévy et al., Eur. Phys. Journ. A25, s01 111 (2005)

[11] B. Bastin et al., Phys. Rev. Lett. 99, 022503 (2007)
[12] F. Sarazin et al., Phys. Rev. Lett. 84, 5062 (2000)

[13] B. Jurado et al., Phys. Lett. B 649, 43 (2007)

[14] A. Gade et al. Phys. Rev. C 71, 051301(R) (2005)

[15] L. Gaudefroy et al., Phys. Rev. Lett. 97, 092501 (2006)

[16] T. R. Werner et al., Nucl. Phys. A597, 327 (1996)

[17] G. A. Lalazissis, D. Vretenar, P. Ring, M.Stoitsov and L. M. Robledo, Phys. Rev. C 60, 014310 (1999)

[18] S. Péru, M. Girod and J. F. Berger, Eur. Phys. Journ. A9, 35 (2000)

[19] R. Rodrígues-Guzmán, J. L. Egido and L. M. Robledo, Phys. Rev. C 65, 024304 (2002)

[20] J. Retamosa, E. Caurier, F. Nowacki and A. Poves, Phys. 
Rev. C 55, 1266 (1997)

[21] D. J. Dean et al., Phys. Rev. C 59, 2474 (1999)

[22] E. Caurier, F. Nowacki and A. Poves, Eur. Phys. Journ. A15, 145 (2002)

[23] E. Caurier, F. Nowacki and A. Poves, Nucl. Phys. A742, $14(2004)$

[24] E. Caurier, G. Martínez-Pinedo, F. Nowacki, A. Poves and A. P. Zuker, Rev. Mod. Phys. 77, 427 (2005)

[25] A.C.C. Villari et al., Nucl. Instrum. Methods B 204, 173 (2003)

[26] S. Ottini-Hustache et al., Nucl. Instrum. Methods A 431, 476 (1999)

[27] Y. Blumenfeld et al., Nucl. Instrum. Methods A 421, 471 (1999)

[28] L. Bianchi et al., Nucl. Instrum. Methods A 276, 509 (1989)

[29] P. Nyborg and O.Skjeggestad, Notes on Phase Space in Kinematics and Multiparticle Systems (Gordon and Breach), 1968

[30] W. Fitz, R Jahr and R. Santo, Nucl. Phys. A114, 392 (1968)

[31] S. Sen et al., Nucl. Phys. A250, 45 (1975)
[32] J. Mrazek et al. Nucl. Phys. A734, E65 (2004)

[33] G. Audi et al. Nucl. Phys. A729, 337 (2003)

[34] P. D. Kunz, University of Colorado, (unpublished)

[35] W. W. Daehnick et al., Phys. Rev. C 21, 2253 (1980)

[36] G. L. Wales and R. C. Johnson, Nucl. Phys. A274, 168 (1976)

[37] R. L. Varner et al., Phys. Rep. 201, 57 (1991)

[38] G. M. Perey and F. G. Perey, At. Data Nucl. Data Tables 17, 1 (1976)

[39] E. Caurier, ANTOINE code, IReS, Strasbourg 1989-2002

[40] E. Caurier and F. Nowacki, Act. Phys. Pol. B 30, 705 (1999)

[41] S. Nummela et al., Phys. Rev. C 63, 044316 (2001)

[42] F. Nowacki and A. Poves, arXiv:0712.2936v1[nucl-th]

[43] J. H. Bjerregaard, O. Hansen and G. Sidenius, Phys. Rev. 138, B 1097 (1965)

[44] P. D. Cottle and K. W. Kemper, Phys. Rev. C 58, 3761 (1998)

[45] C. M. Campbell et al., Phys. Lett. B 652, 169 (2007)

[46] M. Dufour and A. P. Zuker, Phys. Rev. C 54, 1641 (1996) 